Research Paper

\title{
On the application of spectral filters in a Fourier option pricing technique
}

\author{
M. J. Ruijter, ${ }^{1}$ M. Versteegh ${ }^{2}$ and C. W. Oosterlee ${ }^{1,2}$ \\ ${ }^{1}$ Centrum Wiskunde \& Informatica, PO Box 94079, 1090 GB Amsterdam, The Netherlands; \\ emails: marjonruijter@gmail.com, c.w.oosterlee@cwi.nl \\ ${ }^{2}$ Delft University of Technology, Delft Institute of Applied Mathematics, Mekelweg 4, \\ 2628 CD Delft, The Netherlands; email: versteegh@gmail.com
}

(Received 17 May 2013; revised 6 February 2014; accepted 10 March 2014)

\begin{abstract}
When Fourier techniques are applied to specific problems from computational finance with nonsmooth functions, the so-called Gibbs phenomenon may become apparent. This seriously affects the efficiency and accuracy of the numerical results. For example, the variance gamma asset price process gives rise to algebraically decaying Fourier coefficients, resulting in a slowly converging Fourier series. We apply spectral filters to achieve faster convergence. Filtering is carried out in Fourier space; the series coefficients are pre-multiplied by a decreasing function. This does not add any significant computational costs. Tests with different filters show how the algebraic index of convergence is improved.
\end{abstract}

Keywords: Fourier cosine expansion method; spectral filters; European options; variance gamma; portfolio loss distribution; Gibbs phenomenon.

\section{INTRODUCTION}

Fourier techniques have now become well-established in computational finance for efficiently pricing certain financial instruments. For instance, European options, 
certain options with early-exercise features and also exotic options, like Asian, multiasset or barrier options, have been all priced by Fourier techniques. The Fourier techniques belong to the class of numerical integration option pricing methods. They are referred to as "transform methods", because a transformation to the Fourier domain is combined with numerical integration (Boyarchenko and Levendorskii 2011; Carr and Madan 1999; Den Iseger 2006; Lord and Kahl 2007). Transform methods can readily be used for asset price models for which the characteristic function (ie, the Fourier transform of the probability density function) is available. A specific Fourier pricing technique, which we consider here, is the COS method (Fang and Oosterlee 2008, 2009). This pricing method is based on Fourier cosine series expansions. The issues and remedies we address here will, however, be of relevance for other transform methods as well.

As long as the governing probability density function is sufficiently smooth, an exponential error convergence in the number of cosine terms is achieved by the COS method. For certain choices of the asset dynamics, however, the governing probability density function is not smooth everywhere. Smoothness issues are encountered, for example, when we model the asset price by the variance gamma (VG) process (Madan et al 1998). This results in only algebraic convergence for the option price. For hedging purposes, agents are also interested in the option Greeks, which measure risk sensitivities of the option price. Their approximations may even suffer more from nonsmoothness conditions and for these cases it is desirable to find a faster converging method for the Greeks. In the field of risk management we deal with discrete random variables representing individual obligors that may have a default problem. Portfolio loss modeling with small-sized portfolios typically produces stepwise cumulative distribution functions and inaccuracies around the discontinuities may occur.

In general, when Fourier techniques are employed to specific cases with nonsmooth functions, the Gibbs phenomenon may become apparent. This seriously affects the efficiency and accuracy of the financial valuation. The Gibbs phenomenon reflects the difficulty of approximating a discontinuous function by a finite Fourier series. Although the limit of the partial sums represents the original function exactly, in the finite case there is always an overshoot at a jump discontinuity. The width of this overshoot decreases with the number of Fourier terms, but the height of the maximum does not. The Gibbs phenomenon is also related to the principle that the decay of the Fourier coefficients is governed by the smoothness of the function concerned. Functions with a discontinuity in one of the derivatives will have algebraically decaying Fourier coefficients, that result in a slowly converging Fourier series. The local effect of the Gibbs phenomenon gives rise to oscillations near the jumps. However, there is also a global effect: although the error decays away from the jumps, the decay rate is only first order. Thus, the existence of one or more discontinuities drastically reduces 
the convergence rate over the whole domain, and spectral accuracy is lost (Gottlieb and Shu 1997; Tadmor 2007).

The research field dealing with Gibbs oscillations is wide and well-established. An excellent overview into the various improvement techniques is given by Tadmor (2007). We will focus on the use of spectral filters to deal with the Gibbs phenomenon appearing for nonsmooth densities and discrete distribution functions. This is one of the very basic techniques in this field, but we will see that it fits very well to the applications at hand.

Several other techniques have been proposed in the literature to reduce or remove the Gibbs phenomenon. The optimal filter order for a function with discontinuities is an increasing function of the distance to the nearest discontinuity. The idea of adaptive filtering is then to vary the filter order so that it is close to this optimal value. Tadmor (2007); Tanner (2006) and Tadmor and Tanner (2005) describe adaptive filters recovering root-exponential accuracy. This type of filtering is related to superconvergent extraction techniques in finite element methods (van Slingerland et al 2011). However, it is not trivial to efficiently employ an adaptive filter in the context of Fourier option pricing, so we stay with nonadaptive filters here. Mollifiers are a time domain equivalent to filters, in the sense that multiplication by a function in Fourier-space corresponds to a convolution in physical space (which is the basis for mollifiers). Implementation of mollifiers in a Fourier option pricing technique would require reconstruction to the time domain, which is computationally relatively expensive. The same is true for methods like Gegenbauer polynomial reconstruction (see Gottlieb and Shu 1997; Tadmor 2007), digital total variation (DTV) filtering (Chan et al 2001; Sarra 2006) and for the hybrid methods (Glasserman and Ruiz-Mata 2006), where a polynomial reconstruction is used only where needed and filtering is used elsewhere.

We start in Section 2 with Fourier series and Fourier cosine series and explain how we can employ the characteristic function of a random variable to approximate the corresponding density or distribution function. Short introductions to Fourier pricing techniques and the COS method for pricing European options are also given. In Section 3 the convergence of the series and improvements by spectral filters are discussed. Extensive numerical experiments are performed in Section 4. Finally, Section 5 concludes.

\section{FOURIER AND FOURIER COSINE SERIES EXPANSIONS}

The Fourier series of an integrable function $f(y)$ supported on a finite interval $[a, b]$ is defined as (Stein and Shakarchi 2003)

$$
f(y)=\sum_{k=-\infty}^{\infty} \mathcal{F}_{k}^{F} \exp \left(\mathrm{i} k \pi \frac{2 y}{b-a}\right),
$$


with Fourier coefficients given by

$$
\mathscr{F}_{k}^{F}=\frac{1}{b-a} \int_{a}^{b} f(y) \exp \left(-\mathrm{i} k \pi \frac{2 y}{b-a}\right) \mathrm{d} y .
$$

The COS method for pricing European options is based on the Fourier cosine series expansion, which is defined by

$$
f(y)=\sum_{k=0}^{\infty} \mathcal{F}_{k} \cos \left(k \pi \frac{y-a}{b-a}\right),
$$

with Fourier cosine coefficients given by

$$
\mathcal{F}_{k}=\frac{2}{b-a} \int_{a}^{b} f(y) \cos \left(k \pi \frac{y-a}{b-a}\right) \mathrm{d} y .
$$

The prime' in the summation indicates division of the first term by two. These cosine series can be seen as a classical Fourier series of a function $f^{\text {ext }}(y)=f(|y-a|)$, on an extended interval $[2 a-b, b]$, which is mirrored around the midpoint $a$ to make it an even function.

\subsection{Recovery density and distribution function}

In financial option pricing we deal with stochastic asset prices. The corresponding probability density function is usually unknown. However, the characteristic function is often known (Duffie et al 2000) and can be used to approximate the density and distribution function, as we explain in this section. In Section 4.3 we discuss a model for discrete portfolio losses. For example, the loss may be either 0 or 1 . An approximation of the distribution function of a discrete random variable, also by using the characteristic function, is derived here as well.

\subsubsection{Continuous random variable.}

Suppose we have a continuous random variable $X$, with cumulative distribution function $F(\cdot)$ and probability density function $p(\cdot)$. The characteristic function $\varphi(\cdot)$ of $X$ is defined as

$$
\varphi(u):=\mathbb{E}\left[\mathrm{e}^{\mathrm{i} u X}\right]
$$

The density and the characteristic function form a Fourier pair: the characteristic function is the Fourier transform of the density and the density is the inverse Fourier transform of the characteristic function:

$$
\begin{aligned}
& \varphi(u)=\int_{\mathbb{R}} \mathrm{e}^{\mathrm{i} u y} \mathrm{~d} F(y)=\int_{\mathbb{R}} \mathrm{e}^{\mathrm{i} u y} p(y) \mathrm{d} y, \\
& p(y)=\frac{1}{2 \pi} \int_{\mathbb{R}} \mathrm{e}^{-\mathrm{i} u y} \varphi(u) \mathrm{d} u .
\end{aligned}
$$


The Fourier cosine series expansion of the density function reads

$$
p(y)=\sum_{k=0}^{\infty} \mathcal{P}_{k} \cos \left(k \pi \frac{y-a}{b-a}\right),
$$

with Fourier cosine coefficients

$$
\begin{aligned}
\mathcal{P}_{k} & =\frac{2}{b-a} \int_{a}^{b} p(y) \cos \left(k \pi \frac{y-a}{b-a}\right) \mathrm{d} y \\
& =\frac{2}{b-a} \operatorname{Re}\left\{\int_{a}^{b} p(y) \exp \left(\mathrm{i} k \pi \frac{y-a}{b-a}\right) \mathrm{d} y\right\},
\end{aligned}
$$

where $\operatorname{Re}\{\cdot\}$ denotes taking the real part. If the density function $p(y)$ decays rapidly to zero for $y \rightarrow \pm \infty$, the integration range in (2.6a) can be truncated without loss of any significant accuracy:

$$
\varphi(u)=\int_{\mathbb{R}} \mathrm{e}^{\mathrm{i} u y} p(y) \mathrm{d} y \approx \int_{a}^{b} \mathrm{e}^{\mathrm{i} u y} p(y) \mathrm{d} y:=\varphi_{\operatorname{Trunc}}(u),
$$

and therefore the characteristic function can be used to efficiently calculate the Fourier coefficients (Fang and Oosterlee 2008). Combining (2.8) and (2.9) gives us:

$$
\begin{aligned}
\mathcal{P}_{k} & =\frac{2}{b-a} \operatorname{Re}\left\{\varphi_{\operatorname{Trunc}}\left(\frac{k \pi}{b-a}\right) \exp \left(\mathrm{i} k \pi \frac{-a}{b-a}\right)\right\} \\
& \approx \frac{2}{b-a} \operatorname{Re}\left\{\varphi\left(\frac{k \pi}{b-a}\right) \exp \left(\mathrm{i} k \pi \frac{-a}{b-a}\right)\right\} \\
& :=\Phi_{k} .
\end{aligned}
$$

After truncation of the series summation we end up with the approximation

$$
p(y) \approx \sum_{k=0}^{N} \Phi_{k} \cos \left(k \pi \frac{y-a}{b-a}\right) .
$$

The distribution function can now be approximated as follows:

$$
\begin{aligned}
F(y)= & \mathbb{P}(X \leqslant y) \approx \int_{a}^{y} p(t) \mathrm{d} t \approx \sum_{k=0}^{N} \Phi_{k} \int_{a}^{y} \cos \left(k \pi \frac{t-a}{b-a}\right) \mathrm{d} t \\
= & \frac{1}{2} \frac{2}{b-a}(y-a) \\
& +\sum_{k=1}^{N} \frac{2}{k \pi} \operatorname{Re}\left\{\varphi\left(\frac{k \pi}{b-a}\right) \exp \left(\mathrm{i} k \pi \frac{-a}{b-a}\right)\right\} \sin \left(k \pi \frac{y-a}{b-a}\right) .
\end{aligned}
$$


If the interval $[a, b]$ is chosen sufficiently wide, then the series truncation error dominates the error of the approximations (2.11) and (2.12). We refer to Section 3.5 for details about the series truncation error in the COS formula.

\subsubsection{Discrete random variable}

If $X$ is a discrete random variable, then a density function does not exist and we use the following Lévy inversion formula connecting the distribution function, $F(\cdot)$, and characteristic function, $\varphi(\cdot)$. For $y-h, y+h \in C(F)$, with $C(F)$ the continuity set of $F$, there holds (Gut 2005)

$$
\begin{aligned}
\frac{F(y+h)-F(y-h)}{2 h} & =\lim _{T \rightarrow \infty} \frac{1}{2 h} \frac{1}{2 \pi} \int_{-T}^{T} \varphi(u) \frac{\mathrm{e}^{-\mathrm{i} u(y-h)}-\mathrm{e}^{-\mathrm{i} u(y+h)}}{\mathrm{i} u} \mathrm{~d} u \\
& =\lim _{T \rightarrow \infty} \frac{1}{2 \pi} \int_{-T}^{T} \varphi(u) \mathrm{e}^{-\mathrm{i} u y} \frac{\sin u h}{u h} \mathrm{~d} u
\end{aligned}
$$

Suppose $F$ is concentrated on the interval $[a, \infty)$ and $F(a)=0$. Then we get $(h \geqslant 0)$

$$
\begin{aligned}
F(a+h) & =\lim _{T \rightarrow \infty} \frac{1}{\pi} \int_{-T}^{T} \varphi(u) \mathrm{e}^{-\mathrm{i} u a} \frac{\sin u h}{u} \mathrm{~d} u \\
& =\lim _{T \rightarrow \infty} \frac{2}{\pi} \int_{0}^{T} \operatorname{Re}\left\{\varphi(u) \mathrm{e}^{-\mathrm{i} u a}\right\} \frac{\sin u h}{u} \mathrm{~d} u .
\end{aligned}
$$

Numerical integration with step size $\Delta u$ results in the approximation $(y \geqslant a, y \in$ $C(F))$

$$
\begin{aligned}
F(y) & =\lim _{T \rightarrow \infty} \frac{2}{\pi} \int_{0}^{T} \operatorname{Re}\left\{\varphi(u) \mathrm{e}^{-\mathrm{i} u a}\right\} \frac{\sin u(y-a)}{u} \mathrm{~d} u \\
& \approx \frac{1}{2} \frac{2 \Delta u}{\pi}(y-a)+\sum_{k=1}^{N} \frac{2}{k \pi} \operatorname{Re}\left\{\varphi(k \Delta u) \mathrm{e}^{-\mathrm{i} k \Delta u a}\right\} \sin (k \Delta u(y-a)) .
\end{aligned}
$$

For $\Delta u=\pi /(b-a)$ this, in fact, corresponds to the formula for the distribution function of a continuous random variable (2.12).

\subsection{COS method for European options}

The asset price is modeled by a stochastic process and is denoted by $S(t)$. The riskneutral valuation formula for a European option with payoff function $g(\cdot)$ reads

$$
v\left(t_{0}, x\right)=\mathrm{e}^{-r \Delta t} \mathbb{E}_{\mathbb{Q}}\left[g(X(T)) \mid X\left(t_{0}\right)=x\right]=\mathrm{e}^{-r \Delta t} \int_{\mathbb{R}} g(y) p(y \mid x) \mathrm{d} y .
$$


Here $X(t)$ is the state process, which can be any monotone function of the underlying asset price $S(t)$. From here it is taken to be the scaled log-asset price, $X(t):=$ $\log (S(t) / K)$, where $K$ is the option's strike price. $\mathbb{E}_{\mathbb{Q}}$ denotes the expectation under risk-neutral measure $\mathbb{Q} ; \Delta t$ is the difference between the time of maturity $T$ and the initial time $t_{0}=0 ; p(y \mid x)$ is the conditional probability density of $X(T)$, given $X\left(t_{0}\right)=x$, and $r$ is the risk-free interest rate. In other words, the option price is equal to the expected value of its discounted future payoff, under a certain probability measure. Explicit expressions for probability density functions $p(y \mid x)$ encountered in finance are often not known, or involve some mathematical special functions, which make them impractical to calculate. Instead the characteristic function $\varphi(u \mid x)$ corresponding to $p(y \mid x)$ is often known (Duffie et al 2000).

At first Heston (1993) found a closed-form solution for European options with stochastic volatility by means of the Fourier transform. In Carr and Madan (1999), the Fourier transform of the damped payoff function, together with a fast Fourier transform (FFT), was used to evaluate European options under a broad class of models. Fourier methods for Bermudan options were then developed in, among others, O'Sullivan (2005) and Lord et al (2008). For recent developments in pricing exotic options, like Asian and multi-asset options, we refer to Fusai et al (2011), Surkov (2009), Zhang and Oosterlee (2013) and Ruijter and Oosterlee (2012). In this paper we consider a specific Fourier technique, the COS method, which is short for Fourier cosine pricing method. The method is based on the Fourier cosine series expansion of a density function. It can be used to efficiently approximate the expected value of an arbitrary function of random variables. The method was developed in the first place for pricing financial options, like European options (Fang and Oosterlee 2008) and Bermudan options (Fang and Oosterlee 2009). Below we briefly summarize the derivation of the COS formula.

We can replace the density function by its Fourier cosine series expansion,

$$
p(y \mid x)=\sum_{k=0}^{\infty} \mathcal{P}_{k}(x) \cos \left(k \pi \frac{y-a}{b-a}\right),
$$

whose coefficients are approximated by, similarly to (2.10),

$$
\begin{aligned}
\mathcal{P}_{k}(x) & =\frac{2}{b-a} \int_{a}^{b} p(y \mid x) \cos \left(k \pi \frac{y-a}{b-a}\right) \mathrm{d} y \\
& \approx \frac{2}{b-a} \operatorname{Re}\left\{\varphi\left(\frac{k \pi}{b-a} \mid x\right) \exp \left(\mathrm{i} k \pi \frac{-a}{b-a}\right)\right\}:=\Phi_{k}(x) .
\end{aligned}
$$

The Fourier cosine series expansion of the payoff function is given by

$$
g(y)=\sum_{k=0}^{\infty} g_{k} \cos \left(k \pi \frac{y-a}{b-a}\right),
$$


with the coefficients

$$
g_{k}=\frac{2}{b-a} \int_{a}^{b} g(y) \cos \left(k \pi \frac{y-a}{b-a}\right) \mathrm{d} y .
$$

For several payoff functions, including plain vanilla puts and calls, the cosine coefficients $g_{k}$ are available in closed form. Truncating the integration range and substituting the series (2.17) into (2.16), interchanging summation and integration, using definition (2.20) and replacing the coefficients $\mathcal{P}_{k}(x)$ by $\Phi_{k}(x)$ we get a formula for $v\left(t_{0}, x\right)$, written as the product of Fourier cosine coefficients:

$$
v\left(t_{0}, x\right) \approx \frac{b-a}{2} \mathrm{e}^{-r \Delta t} \sum_{k=0}^{\infty} \Phi_{k}(x) g_{k} .
$$

Truncating the series, we obtain the next approximation, the COS pricing formula:

$$
\begin{aligned}
\hat{v}\left(t_{0}, x\right) & :=\mathrm{e}^{-r \Delta t} \sum_{k=0}^{N} \operatorname{Re}\left\{\varphi\left(\frac{k \pi}{b-a} \mid x\right) \exp \left(\mathrm{i} k \pi \frac{-a}{b-a}\right)\right\} g_{k} \\
& =\mathrm{e}^{-r \Delta t} \sum_{k=0}^{N} \operatorname{Re}\left\{\phi\left(\frac{k \pi}{b-a}\right) \exp \left(\mathrm{i} k \pi \frac{x-a}{b-a}\right)\right\} g_{k} .
\end{aligned}
$$

The last equality holds for processes with independent increments, such as Lévy processes, which include the log-versions of geometric Brownian motion, variance gamma and CGMY models. In that case, the characteristic function can be written as a product of $\mathrm{e}^{\mathrm{i} u x}$ and a part independent of $x$, that is $\varphi(u \mid x)=\mathrm{e}^{\mathrm{i} u x} \phi(u)$.

The integration range $[a, b]$ must be chosen carefully to avoid significant errors. An interval which is too small will result in integration range truncation errors, and a too wide interval may give rise to cancelation errors. For now we mention the results given by Fang and Oosterlee (2008), in which a rule of thumb for choosing the integration range is given:

$$
[a, b]:=\left[\kappa_{1}-L \sqrt{\kappa_{2}+\sqrt{\kappa_{4}}}, \kappa_{1}+L \sqrt{\kappa_{2}+\sqrt{\kappa_{4}}}\right], \quad L=10 .
$$

where $\kappa_{1}, \kappa_{2}, \ldots$ are the cumulants of the underlying stochastic process $X(t)$, given in Fang and Oosterlee (2008, Table 11).

\subsubsection{The option Greeks}

The Greeks indicate the sensitivities of the option price with respect to a change in its underlying or model parameters, such as the asset price or the volatility. They are used to hedge the risks in a portfolio. The most well-known Greek parameter is the 
option Delta, $\Delta$, ie, the first derivative of the option price with respect to underlying asset price $S(t)$. Gamma, $\Gamma$, is the second derivative of the option price with respect

to the asset price. By the COS pricing formula (2.22) we naturally find the following approximations $(x=\log (S / K))$,

$$
\begin{aligned}
\Delta & =\frac{\partial v\left(t_{0}, x\right)}{\partial S} \approx \frac{\partial \hat{v}\left(t_{0}, x\right)}{\partial x} \frac{1}{S} \\
& =\mathrm{e}^{-r \Delta t} \sum_{k=0}^{N} \operatorname{Re}\left\{\phi\left(\frac{k \pi}{b-a}\right) \exp \left(\mathrm{i} k \pi \frac{x-a}{b-a}\right) \frac{\mathrm{i} k \pi}{b-a}\right\} g_{k} \frac{1}{S}, \\
\Gamma & =\frac{\partial^{2} v\left(t_{0}, x\right)}{\partial S^{2}} \approx\left(\frac{\partial^{2} \hat{v}\left(t_{0}, x\right)}{\partial x^{2}}-\frac{\partial \hat{v}\left(t_{0}, x\right)}{\partial x}\right) \frac{1}{S^{2}} \\
& =\mathrm{e}^{-r \Delta t} \sum_{k=0}^{N} \operatorname{Re}\left\{\phi\left(\frac{k \pi}{b-a}\right) \exp \left(\mathrm{i} k \pi \frac{x-a}{b-a}\right)\left[\left(\frac{\mathrm{i} k \pi}{b-a}\right)^{2}-\frac{\mathrm{i} k \pi}{b-a}\right]\right\} g_{k} \frac{1}{S^{2}} .
\end{aligned}
$$

\section{CONVERGENCE AND IMPROVEMENTS BY SPECTRAL FILTERS}

In this section, we use, without loss of generality, the interval $[a, b]=[0,2 \pi]$ and we consider the classical Fourier series. As the Fourier cosine series of a function is equivalent to the symmetrically extended version on an extended domain, the theory here also applies to Fourier cosine series.

\subsection{Convergence of Fourier series}

The partial sum of the Fourier series of a function $f(y)$ on $[0,2 \pi]$ is given by

$$
f_{N}(y):=\sum_{|k| \leqslant N} \mathcal{F}_{k}^{F} \mathrm{e}^{\mathrm{i} k y} .
$$

To make statements about the convergence rate we look at pointwise convergence. If $f$ is a continuous periodic function on $[0,2 \pi]$, so $f(0)=f(2 \pi)$, and the Fourier series of $f$ is absolutely convergent, $\sum_{k=-\infty}^{\infty}\left|\mathcal{F}_{k}^{F}\right|<\infty$, then the Fourier series converges uniformly to $f$. For a noncontinuous function there is no uniform convergence and its Fourier series converges to the average of the left- and right-hand limits at a jump discontinuity. In the case that discontinuities of any order are present, the Fourier series expansion only exhibits algebraic convergence. For jump discontinuities, we even encounter zeroth order convergence, which leads to the Gibbs-overshoot.

The speed at which the Fourier coefficients decay depends on the smoothness of the function, as is stated in the following theorem. 
THEOREM 3.1 (Integration-by-parts coefficient bound (Boyd 2001)) If

$$
f(0)=f(2 \pi), f^{(1)}(0)=f^{(1)}(2 \pi), \ldots, f^{(n-2)}(0)=f^{(n-2)}(2 \pi)
$$

and $f^{(n)}(y)$ is integrable, then

$$
\mathcal{F}_{k}^{F} \sim \mathcal{O}\left(|k|^{-n}\right) \text { as }|k| \rightarrow \infty .
$$

Here $f^{(n)}$ denotes the $n$th derivative of $f(y)$. The integrability of $f^{(n)}$ requires that $f(y), f^{(1)}(y), \ldots, f^{(n-2)}(y)$ should be continuous.

The absolute error of truncation of the expansion after $N$ terms is denoted by

$$
E_{\mathrm{Tr}}(N):=\left|f(y)-f_{N}(y)\right| \leqslant \sum_{k=N+1}^{\infty}\left|\mathcal{F}_{k}^{F}+\mathcal{F}_{-k}^{F}\right| .
$$

In general the convergence rate of a Fourier series depends on the smoothness of the function on the expansion interval. We refer to Boyd (2001) for the definitions of algebraically and exponentially converging terms. The following proposition allows us to bound the series truncation error of geometrically and algebraically converging series.

Proposition 3.2 (Last coefficient error estimate (Boyd 2001)) The truncation error is of the same order of magnitude as the last coefficient retained in the truncation for a series with (at least) geometric convergence.

If the series has algebraic convergence index $n>1$, ie, if $a_{k} \sim \mathcal{O}\left(1 / k^{n}\right)$ for large $k$, then

$$
E_{\operatorname{Tr}}(N) \sim \mathcal{O}\left(\left|N a_{N}\right|\right) .
$$

In the numerical experiments in Section 4.1 we will observe that the Fourier coefficients of an $(n-2)$-times continuously differentiable function, $f \in C^{n-2}$, will converge with an algebraic index of convergence $n$ (see Theorem 3.1). According to the proposition above we would expect truncation error $E_{\mathrm{Tr}}(N)$ to decrease with order $\mathcal{O}\left(N^{1-n}\right)$. However, we find a faster convergence, of order $n$. This may be due to the alternating behavior of the series.

\subsection{Spectral filter}

In this section we explain the notion of spectral filters, by which we aim to mitigate oscillations related to the Gibbs phenomenon and achieve faster convergence for problems in financial mathematics. Filtering is carried out in Fourier space and the idea is to pre-multiply the expansion coefficients by a decreasing function in such a way that these decay faster. A properly chosen filter will improve the convergence rate away from discontinuities. The following definition is from Vandeven (1991) and Gottlieb and Shu (1997). 
Definition 3.3 (Fourier space filter of order $p$ ) A real and $C^{\infty}([0,1])$ even function $\hat{s}(\eta)$ is called a filter of order $p$ if

(1) $\hat{s}(0)=1$ and $\hat{s}^{(\ell)}(0)=0,1 \leqslant \ell \leqslant p-1$,

(2) $\hat{s}(\eta)=0$ for $|\eta| \geqslant 1$,

(3) $\hat{s}(\eta) \in C^{p-1}, \eta \in(-\infty, \infty)$.

Conditions (2) and (3) imply $\hat{s}^{(\ell)}(1)=0,0 \leqslant \ell \leqslant p-1$.

The filtered partial sum of a Fourier series is simply defined by

$$
f_{N}^{\hat{s}}(y)=\sum_{|k| \leqslant N} \hat{s}(k / N) \mathcal{F}_{k}^{F} \mathrm{e}^{\mathrm{i} k y} .
$$

We can rewrite this as a convolution in physical space:

$$
f_{N}^{\hat{s}}(y)=\frac{1}{2 \pi} \int_{0}^{2 \pi} s(y-t) f(t) \mathrm{d} t,
$$

with

$$
s(x)=\sum_{|k| \leqslant \infty} \hat{s}(k / N) \mathrm{e}^{\mathrm{i} k x}, \quad x \in[0,2 \pi] .
$$

Note that $\hat{s}(k / N)=0$ for $|k|>N$. A filter is a continuous function which only modifies high frequency modes, not the low modes. Filtering may remove the Gibbs phenomenon from a discontinuity, the error depends on the distance to the discontinuity, as we will confirm in Section 3.3. Since the approximation will be smoothened, convergence in the vicinity of a "jump" will not improve. Filtering does not affect the total mass of the resulting approximation (which should be 1 for a probability density), since the first coefficient is never altered. To be precise,

$$
\begin{aligned}
\int_{0}^{2 \pi} f_{N}^{\hat{s}}(y) \mathrm{d} y & =\sum_{|k| \leqslant N} \hat{s}(k / N) \mathcal{F}_{k}^{F} \int_{0}^{2 \pi} \mathrm{e}^{\mathrm{i} k y} \mathrm{~d} y \\
& =\hat{s}(0 / N) \mathcal{F}_{0}^{F} 2 \pi=\int_{0}^{2 \pi} f(y) \mathrm{d} y .
\end{aligned}
$$

\subsubsection{Examples of spectral filters}

The following filters are well-known from the literature (Gottlieb and Shu 1997; Hesthaven et al 2007; Vandeven 1991).

- Fejér filter (Fejér 1900): $\hat{s}(\eta)=1-|\eta|$, with order $p=1$.

- Lanczos filter (Lanczos 1956): $\hat{s}(\eta)=\sin (\pi \eta) /(\pi \eta)$, with order $p=1$.

- Raised cosine filter: $\hat{s}(\eta)=\frac{1}{2}(1+\cos (\pi \eta))$, with order $p=2$. 
The following general $p$ th order spectral filters also exist.

- Exponential filter (Gottlieb and Shu 1997): $\hat{s}(\eta)=\exp \left(-\alpha \eta^{p}\right)$, where $p$ must be even and $\alpha=-\log \varepsilon_{m}$, with $\varepsilon_{m}$ the machine epsilon.

- Vandeven filter (Peyret 2002; Vandeven 1991):

$$
\hat{s}(\eta)=1-\frac{(2 p-1) !}{((p-1) !)^{2}} \int_{0}^{|\eta|} t^{p-1}(1-t)^{p-1} \mathrm{~d} t .
$$

- Erfc-Log filter (Boyd 1996): Boyd showed that the Vandeven filter can be approximated quite accurately by an analytic function which satisfies all conditions, ie, by the Erfc-Log filter:

$$
\hat{s}(\eta)=\frac{1}{2} \operatorname{erfc}\left(2 \sqrt{p}\left(|\eta|-\frac{1}{2}\right) \sqrt{\frac{-\log \left(1-4(|\eta|-1 / 2)^{2}\right)}{4(|\eta|-1 / 2)^{2}}}\right),
$$

where $\operatorname{erfc}(\cdot)$ is the complimentary Gauss error function.

\subsection{Convergence and error analysis}

A higher-order filter modifies the original function in smooth regions away from a discontinuity and high-order accuracy is desirable away from a discontinuity. Loworder filtering is however desirable close to a discontinuity, because higher $p$ values then give rise to a highly oscillatory filtered function $f_{N}^{\hat{s}}$. The following theorem gives a bound on the error. It can be extended in a straightforward way to a function with more points of discontinuity $\xi_{m}$.

THEOREM 3.4 (Gottlieb and Shu 1997; Hesthaven et al 2007) Let $f(y)$ be a piecewise $C^{p}([0,2 \pi])$ function with one point of discontinuity $\xi$. Let $\hat{s}(k / N)$ be a filter of order $p$. Now let $y$ be a point in $[0,2 \pi]$ and define

$$
d(y):=\min _{k=-1,0,1}|y-\xi+2 k \pi| .
$$

Then (if $y \neq \xi$ ),

$$
\begin{aligned}
& \left|f_{N}^{\hat{s}}(y)-f(y)\right| \\
& \quad=\left|\frac{1}{2 \pi} \sum_{\ell=0}^{p-1} s_{\ell+1}(d(y))\left(f^{(\ell)}\left(\xi^{+}\right)-f^{(\ell)}\left(\xi^{-}\right)\right)+\frac{1}{2 \pi} \int_{0}^{2 \pi} s_{p}(y-t) f^{(p)}(t) \mathrm{d} t\right| \\
& \quad \leqslant c N^{1-p} d(y)^{1-p} \mathcal{K}(f)+c N^{1 / 2-p}\left\|f^{(p)}\right\|_{L^{2}},
\end{aligned}
$$


where

$$
\begin{aligned}
\mathcal{K}(f) & =\sum_{\ell=0}^{p-1} d(y)^{\ell}\left(f^{(\ell)}\left(\xi^{+}\right)-f^{(\ell)}\left(\xi^{-}\right)\right) \int_{-\infty}^{\infty}\left|G_{\ell}^{(p-\ell)}(\eta)\right| \mathrm{d} \eta \\
G_{\ell}(\eta) & =\frac{\hat{s}(\eta)-1}{\eta^{\ell}}
\end{aligned}
$$

$c$ is a constant independent of $f$ and $N$, and

$$
s_{0}(x)=s(x), \quad s_{\ell}^{\prime}=s_{\ell-1}, \quad \ell \geqslant 1, \quad \int_{0}^{2 \pi} s_{\ell}(t) \mathrm{d} t=0, \quad \ell \geqslant 1
$$

The error bound decreases with $d(y)$, ie, with the distance to the discontinuity. The filter order determines the rate at which the error remaining after filtering decays. If we have $f \notin C^{p-1}$, ie, if $f(y)$ has a jump discontinuity at one or more points of order smaller than, or equal to, $p-1$, the following estimate holds: $\left|f_{N}^{\hat{s}}(y)-f(y)\right| \sim$ $\mathcal{O}\left(N^{1-p}\right)$. If $f \in C^{p-1}$, ie, if $f(y)$ is smooth in the sense of possessing at least $p-1$ continuous derivatives, then $\left|f_{N}^{\hat{s}}(y)-f(y)\right| \sim \mathcal{O}\left(N^{(1 / 2)-p}\right){ }^{1}$

In the numerical experiments in Section 4.1 we will observe a somewhat faster convergence than prescribed by Theorem 3.4. This can be explained by the following observations. For the first part in (3.11) the authors in Vandeven (1991) prove by induction that

$$
s_{\ell}(x) \sim \mathcal{O}\left(N^{1-p}\right), \quad x \in(0,2 \pi), 0 \leqslant \ell \leqslant p .
$$

Table 1 on the next page shows the order of convergence for $s_{0}$ and $s_{1}$ that we observed by numerical experiments. We tested six different filters: the Fejér filter, the Lanczos filter, the raised cosine filter, the exponential filter, the Vandeven filter and the ErfcLog filter, as described in Section 3.2. We also used different filter orders. Here "exp" denotes exponential convergence. The algebraic index of convergence given by (3.14) is thus not strict and can be higher than order $p$.

For the second part in (3.11) the authors in Vandeven (1991) and Gottlieb and Shu (1997) use the inequalities

$$
\left|\frac{1}{2 \pi} \int_{0}^{2 \pi} s_{\ell}(y-t) f^{(\ell)}(t) \mathrm{d} t\right| \leqslant \frac{1}{2 \pi} \sqrt{\int_{0}^{2 \pi} s_{\ell}^{2}(y-t) \mathrm{d} t} \sqrt{\int_{0}^{2 \pi}\left(f^{(\ell)}(t)\right)^{2} \mathrm{~d} t}
$$

\footnotetext{
${ }^{1}$ The Euler-accelerated partial sum (Boyd 2011) gives a geometric error, ie, an error of order $\mathcal{O}(\exp (-\mu(y) N))$. However, we are not able to obtain results for $N>1024$ as the computation of the filter is then limited by our double precision computations. Besides, the computational costs of this filter are about 100 times as high as the exponential filter, for our numerical examples.
} 
TABLE 1 Algebraic index of convergence $(x \in(0,2 \pi))$.

\begin{tabular}{lcc}
\hline Filter & $\boldsymbol{s}_{0}(\boldsymbol{x})$ & $\boldsymbol{s}_{1}(\boldsymbol{x})$ \\
\hline Fejér & 1 & 1 \\
Lanczos & 1 & 2 \\
Raised cosine & 2 & 3 \\
\hline Exponential $(p=2,4,6)$ & $\exp$ & $\exp$ \\
Vandeven $(p=1,3,5)$ & $p$ & $p$ \\
Vandeven $(p=2,4)$ & $p$ & $p+1$ \\
Erfc-Log $(p=1,2,3,4,5)$ & $p$ & $p$ \\
\hline
\end{tabular}

and find the upper bound

$$
\left|\frac{1}{2 \pi} \int_{0}^{2 \pi} s_{\ell}(y-t) f^{(\ell)}(t) \mathrm{d} t\right| \leqslant \sim \mathcal{O}\left(N^{1 / 2-\ell}\right) .
$$

However, for the filters in Table 1 we observe by our numerical computations that

$$
\left|\frac{1}{2 \pi} \int_{0}^{2 \pi} s_{\ell}(y-t) f^{(\ell)}(t) \mathrm{d} t\right| \leqslant \sim \mathcal{O}\left(N^{\max (-p, 1 / 2-\ell)}\right), \quad 1 \leqslant \ell \leqslant 10 .
$$

\subsection{Filtering and the COS method}

One of the reasons why the COS method is highly efficient is because pricing formula (2.22) works directly with the coefficients, without a priori recovery of the functions $p(y \mid x)$ or $g(y)$. Spectral filters work strictly in the Fourier domain and therefore they can be used directly in the COS pricing formula. Once a suitable filter and order have been chosen one can multiply the Fourier cosine coefficients by a factor $\hat{s}(k / N)$

and work with the COS method as before. This simply gives us the filter-COS pricing formula, as follows:

$$
v\left(t_{0}, x\right) \approx \mathrm{e}^{-r \Delta t} \sum_{k=0}^{N} \hat{s}\left(\frac{k}{N}\right) \operatorname{Re}\left\{\phi\left(\frac{k \pi}{b-a}\right) \exp \left(\mathrm{i} k \pi \frac{x-a}{b-a}\right)\right\} g_{k},
$$

where $\hat{s}$ can be any nonadaptive filter. It does not add significant computational costs. Unfortunately, the same does not hold for the adaptive filters (Boyd 2011; Tadmor and Tanner 2005; Tanner 2006) because, if we vary coefficients depending on position, we can no longer use the substitution of (2.20) which leads to the COS method. 


\subsection{Error analysis COS method and filter-COS method}

\subsubsection{Without filtering}

The error of the COS formula without filtering terms is composed of three parts: the integration range truncation error, the series truncation error and the error related to approximating $\mathcal{P}_{k}$ by $\Phi_{k}$. A detailed error analysis was given in Fang and Oosterlee (2008), where it was shown that if $p(y \mid x) \in C^{\infty}$, with a properly chosen truncation range $[a, b]$, then the COS method exhibits exponential convergence. Both a density function and payoff function with a discontinuity in one of their derivatives, results in an algebraic convergence. If the computational domain $[a, b]$ is chosen sufficiently wide, then the so-called series truncation error $E_{\mathrm{Tr}}^{\operatorname{COS}}\left(t_{0}, x ; N\right)$, ie,

$$
\begin{aligned}
E_{\operatorname{Tr}}^{\mathrm{COS}}\left(t_{0}, x ; N\right): & =\mathrm{e}^{-r \Delta t} \frac{b-a}{2} \sum_{k=N+1}^{\infty} \Phi_{k}(x) g_{k} \\
& =\mathrm{e}^{-r \Delta t} \int_{a}^{b} g(y)\left(p(y \mid x)-p_{N}(y \mid x)\right) \mathrm{d} y,
\end{aligned}
$$

dominates the total error. Here $p_{N}(y \mid x)$ denotes the truncated Fourier cosine series of the density on $[a, b]$. The series truncation error depends on the smoothness of underlying probability density function $p(y \mid x)$ and payoff function $g(y)$. With (3.19) it follows that if either $\Phi_{k}$ or $g_{k}$ decreases exponentially, then the error has exponential convergence in $N$. If, however, both decay algebraically, then we end up with algebraic convergence. If $\Phi_{k}(x)$ and $g_{k}$ have algebraic index of decay $n_{\varphi}(x)$ and $n_{g}$, respectively, then Proposition 3.2 gives

$$
E_{\operatorname{Tr}}^{\operatorname{COS}}\left(t_{0}, x ; N\right) \sim \mathcal{O}\left(N^{-n_{\varphi}(x)-n_{g}+1}\right)
$$

In the numerical examples we will observe convergence rates

$$
E_{\operatorname{Tr}}^{\operatorname{CoS}}\left(t_{0}, x ; N\right) \sim \mathcal{O}\left(N^{-n_{\varphi}(x)-n_{g}}\right),
$$

which is probably due to the alternating behavior of the series.

Payoff functions, like puts and calls, are in general nonsmooth, which is the reason for slowly decreasing Fourier coefficients. Asset prices modeled by geometric Brownian motion, jump-diffusion or the Heston model lead to exponential decay of the coefficients $\Phi_{k}$, resulting in an exponentially converging COS formula. However, the density functions of the variance gamma and CGMY models may be nonsmooth. Together with a nonsmooth payoff function this will result in rather slow algebraic error convergence in $N$. Applying an appropriate spectral filter will improve the convergence rate. 


\subsubsection{With filtering}

Applying spectral filter $\hat{s}$ in the COS formula leads to the following relation,

$$
\begin{aligned}
E_{\mathrm{Tr}}^{\text {filter-COS }}\left(t_{0}, x ; N\right) & \\
& :=\mathrm{e}^{-r \Delta t} \frac{b-a}{2} \sum_{k=0}^{N}(1-\hat{s}(k / N)) \Phi_{k}(x) g_{k}+\mathrm{e}^{-r \Delta t} \frac{b-a}{2} \sum_{k=N+1}^{\infty} \Phi_{k}(x) g_{k} \\
& =\mathrm{e}^{-r \Delta t} \int_{a}^{b} g(y)\left(p(y \mid x)-p_{N}^{\hat{s}}(y \mid x)\right) \mathrm{d} y
\end{aligned}
$$

The absolute value can be bounded by

$$
\begin{aligned}
& \left|E_{\operatorname{Tr}}^{\text {filter-COS }}\left(t_{0}, x ; N\right)\right| \\
& \quad \leqslant \mathrm{e}^{-r \Delta t} \int_{a}^{b}|g(y)|\left|p(y \mid x)-p_{N}^{\hat{s}}(y \mid x)\right| \mathrm{d} y=\mathcal{O}\left(N^{-n_{p}^{\hat{s}}(x)}\right) .
\end{aligned}
$$

Here, $n_{p}^{\hat{s}}(x)$ denotes the algebraic index of convergence of the error $p(y \mid x)-$ $p_{N}^{\hat{s}}(y \mid x)$ of the filtered partial sum, which is discussed in Section 3.3 (Theorem 3.4). The absolute error depends on the distance to the discontinuity of the density function and may be larger for strike prices $K$ close to the discontinuity. Only at the discontinuity the error will not improve, but we integrate over the whole interval $[a, b]$. We observe higher absolute errors in the option value for strikes near the discontinuity of the density function, but appropriate filters will improve the convergence rate. The filter-COS formula is beneficial especially when the number of terms $N$ increases.

\subsubsection{Option Greeks}

Computing the option Greeks was briefly described in Section 2.2.1. The error of the COS formula for the option Delta, without filtering, is given by

$$
\begin{aligned}
E_{\mathrm{Tr}}^{\operatorname{COS}-\Delta}\left(t_{0}, x ; N\right) & :=\mathrm{e}^{-r \Delta t} \frac{b-a}{2} \sum_{k=N+1}^{\infty} \frac{\partial \Phi_{k}(x)}{\partial x} \xi_{k} \frac{1}{S}, \\
\frac{\partial \Phi_{k}(x)}{\partial x} & =\frac{2}{b-a} \operatorname{Re}\left\{\phi\left(\frac{k \pi}{b-a}\right) \exp \left(\mathrm{i} k \pi \frac{x-a}{b-a}\right) \frac{\mathrm{i} k \pi}{b-a}\right\} .
\end{aligned}
$$

As the coefficients for the Greeks are multiplied by factors $i k \pi /(b-a)$, the algebraic index of convergence is reduced by 1 . So, finding a faster converging method becomes even more beneficial for the option Greeks. With a filter-COS formula for the option Greeks we achieve the same convergence rates as for the option prices. 


\section{NUMERICAL EXAMPLES}

In this section we discuss several numerical experiments supporting the insights from earlier sections. MATLAB 7.11.0 R2010b is used for the computations, with double precision. We start in Section 4.1 with three basic test functions $f(y)$, representing the option payoff or probability density features. Subsequently, in Section 4.2.1 the density recovery of the $\mathrm{VG}$ process is studied. Convergence of option prices, computed by the filter-COS method, is discussed in Section 4.2.2 for European-style options and in Section 4.2.3 for Bermudan-style options. An example from portfolio loss modeling, resulting in a staircase distribution function, is presented in Section 4.3.

\subsection{Convergence test functions}

We perform tests with three different functions $f(y)$ and different filters. The test functions are shown in Figure 1 on the next page, with $[a, b]=[0,2 \pi]$. Function A represents a block function with two jump discontinuities, function $\mathrm{B}$ is smooth and function $\mathrm{C}$ has a discontinuity in the first derivative.

Part (a) of Figure 2 on page 93 shows function A (gray line) and its Fourier series approximation without (dashed gray line) and with (black line) an exponential filter of order $p=4(N=32)$. The middle plot gives the corresponding error. At the jump discontinuities the limit converges to the average of the values of the function at either side of the jump. In part (b) we display the convergence of the error at the point $y=\pi$, for increasing values of $N$ (log-log plot). The local effect of the Gibbs phenomenon gives rise to oscillations near the jumps and the partial sum does not converge at the jump, thereby resulting in a lack of uniform convergence. However, there is also a global effect: although the error decays away from the jumps, the algebraic pointwise convergence is only first order, $\mathcal{O}(1 / N)$. The filter improves the error significantly, especially away from the jump discontinuities. The error at point $y=\pi$ decreases exponentially in $N$ due to the usage of the filter.

To recover the functions, we test the performance of six filters, ie, the Fejér filter, the Lanczos filter, the raised cosine filter, the exponential filter, the Vandeven filter and the Erfc-Log filter (see Section 3.2). We also employ different filter orders. Table 2 on page 94 presents the algebraic index of convergence observed for the three test functions. Here "exp" denotes exponential convergence. With two numbers, eg, "2, 4", convergence is order $\mathcal{O}\left(N^{-2}\right)$ on $[0, \pi]$ and $\mathcal{O}\left(N^{-4}\right)$ on $[\pi, 2 \pi]$. An asterisk $(*)$ in the table indicates that the order of convergence was not clearly measurable in our numerical experiments.

Based on the error analysis of Theorem 3.4 and our observations in Section 3.3 we can explain the numbers in Table 2 on page 94 as follows. 
FIGURE 1 Three test functions $f(y)$.
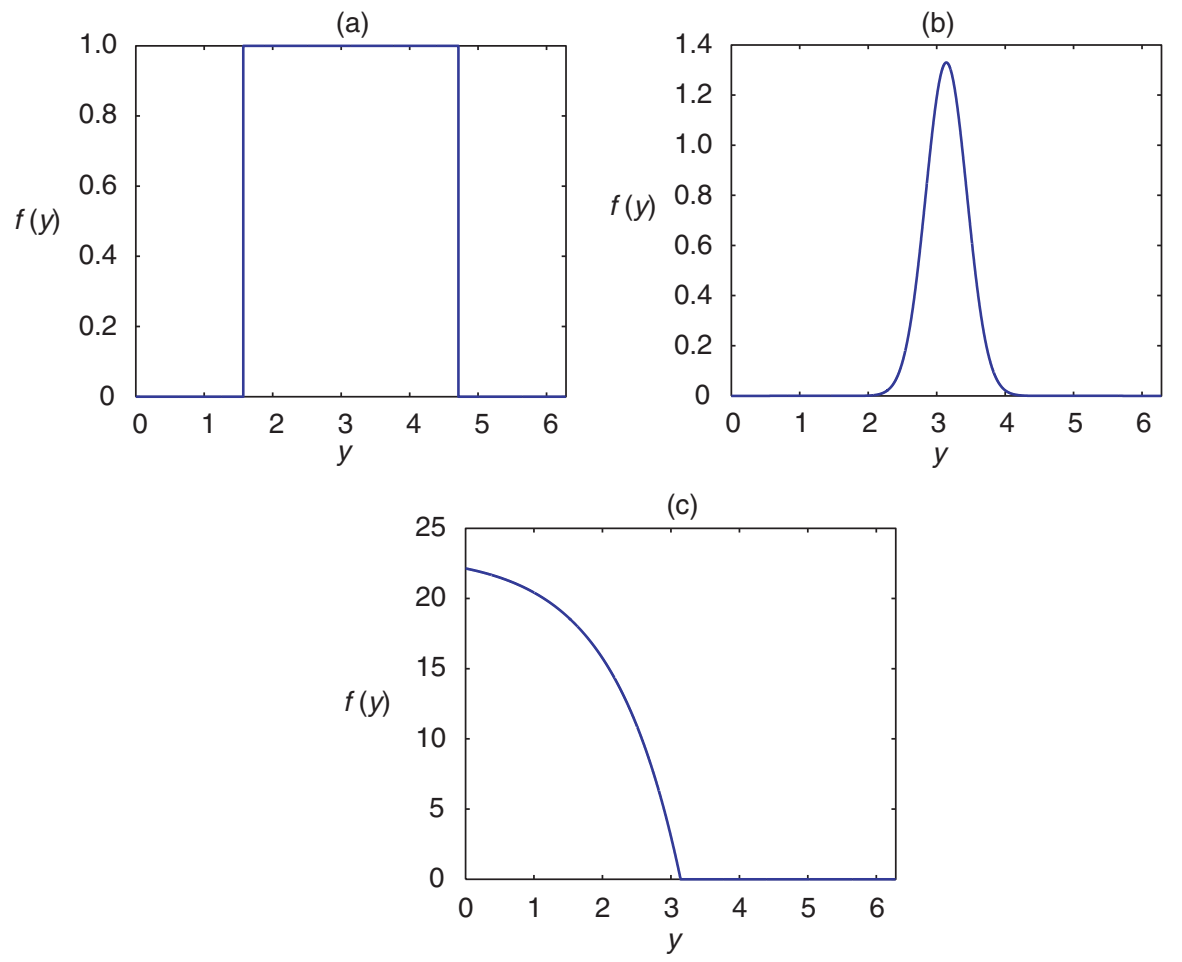

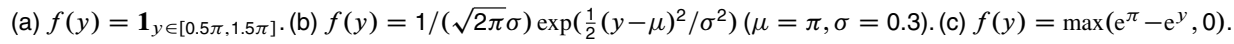

For function A we have

$$
\begin{aligned}
& \left|f_{N}^{\hat{s}}(y)-f(y)\right| \\
& \quad=\sum_{m} \frac{1}{2 \pi} s_{1}\left(d_{m}(y)\right)\left(f\left(\xi_{m}^{+}\right)-f\left(\xi_{m}^{-}\right)\right)+\frac{1}{2 \pi} \int_{0}^{2 \pi} s_{1}(y-t) f^{(1)}(t) \mathrm{d} t \\
& \quad=\sum_{m} \frac{1}{2 \pi} s_{1}\left(d_{m}(y)\right)\left(f\left(\xi_{m}^{+}\right)-f\left(\xi_{m}^{-}\right)\right) .
\end{aligned}
$$

The observed decay rates of $s_{1}$ are given in Table 1 on page 88 and they correspond to the results in the second column in Table 2 on page 94.

Function B is approximately smooth, so that

$$
\left|f_{N}^{\hat{s}}(y)-f(y)\right|=\frac{1}{2 \pi} \int_{0}^{2 \pi} s_{q}(y-t) f^{(q)}(t) \mathrm{d} t, \quad \forall q \geqslant 1 .
$$


FIGURE 2 Recovery $f(y)$ (function A) without and with filter (exponential, order $p=4$ ) $(N=32)$.
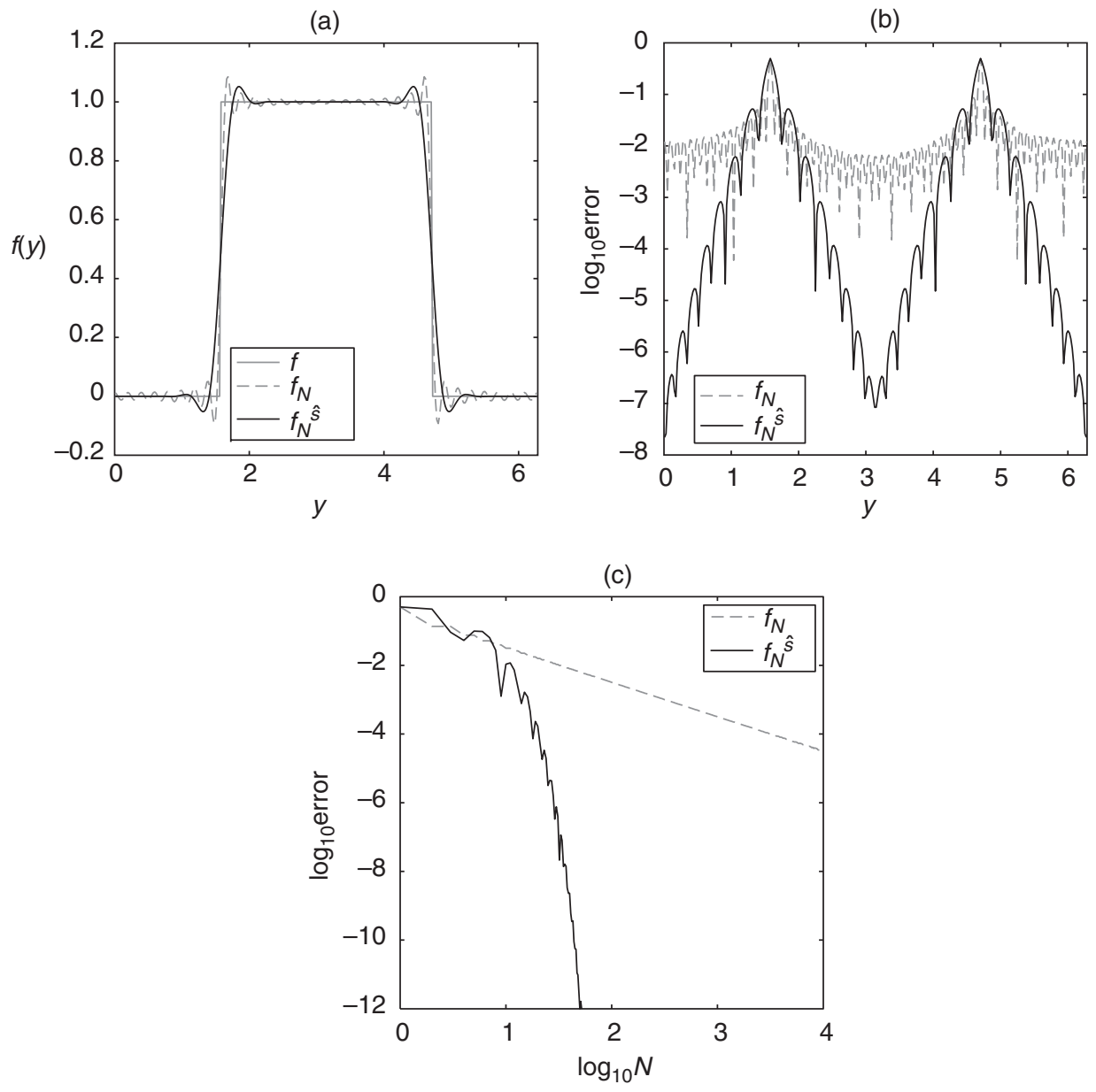

Following the arguments in Section 3.3 we expect

$$
\frac{1}{2 \pi} \int_{0}^{2 \pi} s_{q}(y-t) f^{(q)}(t) \mathrm{d} t \sim \mathcal{O}\left(N^{\max (-p, 1 / 2-q)}\right) .
$$

This gives us the convergence order $\mathcal{O}\left(N^{-p}\right)$, as observed.

The error of function $\mathrm{C}$ corresponds to the error of function B for $y \in[0, \pi]$. And the error corresponds to the error of function A for $y \in[\pi, 2 \pi]$. 
TABLE 2 Algebraic index of convergence test functions.

\begin{tabular}{lccc}
\hline Filter & Function A & Function B & Function C \\
\hline No filter & 1 & $\exp$ & 1 \\
\hline Fejér & 1 & 1 & 1 \\
Lanczos & 2 & 2 & 2 \\
Raised cosine & 3 & 2 & 2,3 \\
\hline Exponential $(p=2)$ & $\exp$ & 2 & $2, \exp$ \\
Exponential $(p=4)$ & $\exp$ & 4 & $4, \exp$ \\
Exponential $(p=6)$ & $\exp$ & 6 & $*, \exp$ \\
Vandeven $(p=1,3,5)$ & $p$ & $p$ & $p$ \\
Vandeven $(p=2,4)$ & $p+1$ & $p$ & $p, p+1$ \\
Erfc-Log $(p=1,2,3,4,5)$ & $p$ & $p$ & $p$ \\
\hline
\end{tabular}

\subsection{The variance gamma process}

In this section, we discuss various applications of the COS method in the context of the VG process (Madan et al 1998; Schoutens 2003). We start with the accurate and efficient recovery of the VG density. Then European and Bermudan option prices are analyzed.

\subsubsection{Variance gamma density recovery}

In the case of modeling asset prices by a fat-tailed density function, the exponential VG jump process can be applied. The VG process is obtained by evaluating a Brownian motion with drift $\theta$ and volatility $\sigma$ at a random time given by a gamma process $\gamma(t)$ with mean rate one and variance rate $v$ (Madan et al 1998; Schoutens 2003):

$$
L_{\mathrm{VG}}(t)=\theta \gamma(t)+\sigma W(\gamma(t)) .
$$

The risk-neutral asset price is then defined as $S(t)=S\left(t_{0}\right) \mathrm{e}^{(r+w) \Delta t} \mathrm{e}^{L_{\mathrm{VG}}(t)}, w=$ $1 / v \log \left(1-\theta v-\frac{1}{2} \sigma^{2} v\right)$ (Fang and Oosterlee 2009). The VG process is of bounded variation, has independent increments and is defined by an infinite arrival of jumps. The VG density can be characterized by a fat tail: it is suitable to model phenomena where small and relatively large asset values are more probable than would be the case for the lognormal distribution. The characteristic function, $\varphi(u \mid x)=\mathbb{E}\left[\mathrm{e}^{\mathrm{i} u X(T)} \mid\right.$ $\left.X\left(t_{0}\right)=x\right]=\mathrm{e}^{\mathrm{i} u x+\mathrm{i} u(r+w) \Delta t} \phi_{\mathrm{VG}}(u)$, is given by Schoutens (2003) and Madan et al (1998), with

$$
\phi_{\mathrm{VG}}(u)=\left(1-\mathrm{i} u \theta v+\frac{1}{2} \sigma^{2} v u^{2}\right)^{-\Delta t / v} \sim \mathcal{O}\left(u^{-2 \Delta t / v}\right) .
$$


FIGURE 3 VG density and error for $T=0.1(N=128)$.
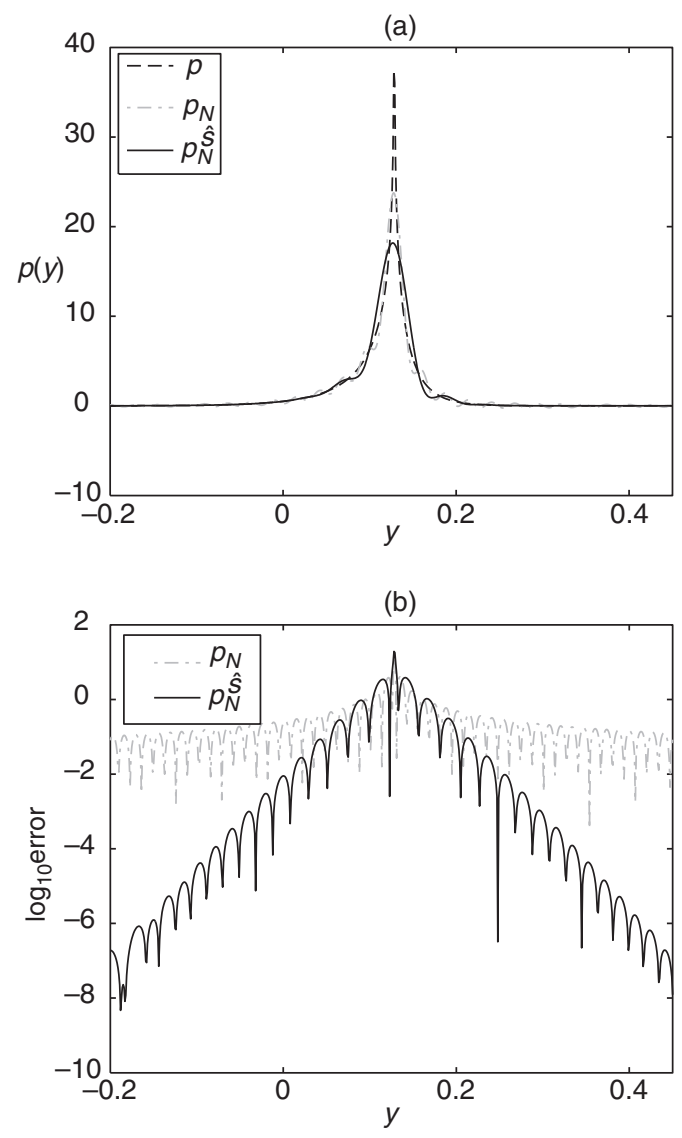

In Madan et al (1998), the following expression for the VG density function was derived:

$p_{\mathrm{VG}}(y)=\int_{0}^{\infty} \frac{1}{\sigma \sqrt{2 \pi z}} \exp \left(-\frac{(y-\theta z)^{2}}{2 \sigma^{2} z}\right) \frac{z^{\Delta t / v-1} \exp (-z / v)}{v^{\Delta t / v} \Gamma(\Delta t / v)} \mathrm{d} z$

(with $\Gamma$ the Gamma function).

It is computationally rather expensive to evaluate (4.6) at each point in the domain of interest. The smoothness of the density function depends on its parameters, or, to be more precise, with higher values of $\Delta t / v$ a larger number of derivatives exists. 
TABLE 3 Algebraic index of convergence VG density recovery with three values of $T$.

\begin{tabular}{lccc}
\hline Filter & $\boldsymbol{T}=\mathbf{0 . 0 2 5}$ & $\boldsymbol{T}=\mathbf{0 . 1}$ & $\boldsymbol{T}=\mathbf{1}$ \\
\hline No filter & 0.25 & 1 & 10 \\
\hline Fejér & 1 & 1 & 1 \\
Lanczos & 2 & 2 & 2 \\
Raised cosine & 2 & 2 & 2 \\
\hline Exponential $(p=2,4)$ & $p$ & $p$ & $p$ \\
Exponential $(p=6)$ & $*$ & 6 & 6 \\
Vandeven $(p=1,2,3,4,5)$ & $p$ & $p$ & $p$ \\
Erfc-Log $(p=1,2,3,4,5)$ & $p$ & $p$ & $p$ \\
\hline
\end{tabular}

The parameters used for our tests here are the same as in Fang and Oosterlee (2008), ie,

$$
\begin{array}{ccc}
K=90, & S(0)=100, & r=0.1 \\
\sigma=0.12, & \theta=-0.14, & v=0.2 .
\end{array}
$$

Figure 3 on the preceding page shows the VG density for terminal time $T=0.1$ (black dashed line). For small values of $T$ the peak in the density gets really sharp and is difficult to approximate accurately by a Fourier cosine series. For increasing $\nu$-values the peak sharpens, while increasing $\sigma$-values result in a smaller peak and wider tails, without altering the smoothness properties of the function around the peak significantly.

Figure 3 also shows the approximations by Fourier cosine series, ie, (2.11), with and without exponential filter (order $p=6$ ). The characteristic function exhibits an algebraic decay with order $2 \Delta t / v$, giving rise to an algebraic decay of the Fourier coefficients and therefore slow convergence of the COS method, especially for the densities with small time interval $\Delta t$. Filtering works well away from the peak in the VG density, but right at the peak the approximation becomes somewhat worse. Note that for larger $T$-values, like for $T=1$, the function is sufficiently smooth and addition of the filter does not improve its already accurate approximation.

We evaluate the performance of the filters to recover the VG density. The six different filters are used and we experiment with different filter orders. We find that lower order filters flatten the sharp peak too much. Table 3 shows the measured algebraic index of convergence for the density recovery. We observe a significant improvement for $T=0.025$ and $T=0.1$, whereas for $T=1$ the "no filter case" is superior. 
TABLE 4 Reference values European options.

\begin{tabular}{lccrrrrr}
\hline & $\overbrace{\text { Put }}^{T=0}$ & Digital & Put & Digital & $\overbrace{\text { Put }}$ & Digital \\
\hline Option value $v\left(t_{0}, x\right)$ & 0.02435 & 89.1883 & 0.09819 & 86.9759 & 0.53472 & 74.7855 \\
Delta $\Delta$ & -0.50629 & 12.8417 & -1.82737 & 40.6550 & -5.50365 & 63.1902 \\
Gamma $\Gamma$ & 11.5565 & -313.402 & 36.5895 & -843.107 & 56.8712 & -581.247 \\
\hline
\end{tabular}

\subsubsection{European options and Greeks under variance gamma}

We investigate here the convergence of the COS and the filter-COS method for pricing European-style options. The payoff function of a put reads $g(S)=\max (K-S, 0)$. For a digital (or binary) call option the payoff is either one or zero, with payoff function $g(S)=\mathbf{1}_{S \geqslant K}$. Reference values for the experiments are obtained by selecting an accurate filter and a very large number of terms in the series expansions (see Table 4). The convergence of the COS method without filtering is of order $2 \Delta t / v+2$ for put options and $2 \Delta t / v+1$ for digital options. The algebraic index of convergence for the Greek Delta $\Delta$ (2.24a), without filtering, is one order lower and for Gamma $\Gamma$ (2.24b) it is two orders lower, see Table 5 on the next page. The absolute value of the series terms gives us $\mathcal{O}\left(1 / k^{2 \Delta t / v+1-2}\right)$ convergence for the Gamma $\Gamma$ of a digital option. So, the algebraic index of convergence is even nonpositive for expiration times $T=0.1$ and $T=0.025$ and the series does not converge ("Div"), because of the Cauchy convergence criterion. The intuition behind this is that for very short expiration times, the $\Gamma$ converges to the Dirac delta function.

Table 6 on the next page shows the observed order of convergence of the European option with different spectral filters. Similar convergence results are obtained for the Greeks $\Delta$ and $\Gamma$.

We tend to prefer the exponential filter to the Vandeven and Erfc-Log filters. Its implementation is easiest and fastest, although the other filters are not significantly more time-consuming. Furthermore, we observe an exponential convergence for step functions with the exponential filter (see Figure 2 on page 93 and Table 2 on page 94), which is advantageous for recovery of a distribution function for discrete random variables. Therefore, we focus on the exponential filter in the remainder of this paper.

For asset price processes with independent increments, like the VG model, we can employ the filter-COS method to compute option values for multiple strike prices simultaneously. For example, for $K \in[80,120]$ we obtain the same convergence results. However, we observe higher absolute errors in the option values for strike prices near the peak value in the VG density function. This can be explained by the smoothing of the peak by a filter. 
TABLE 5 Algebraic index of convergence for European options (no filter).

\begin{tabular}{|c|c|c|c|c|c|c|}
\hline & \multicolumn{2}{|c|}{$T=0.025$} & \multicolumn{2}{|c|}{$T=0.1$} & \multicolumn{2}{|c|}{$T=1$} \\
\hline & Put & Digital & Put & Digital & Put & Digital \\
\hline Option value $v\left(t_{0}, x\right)$ & 2.25 & 1.25 & 3 & 2 & 12 & 11 \\
\hline Delta $\Delta$ & 1.25 & 0.25 & 2 & 1 & 11 & 10 \\
\hline Gamma $\Gamma$ & 0.25 & Div & 1 & Div & 10 & 9 \\
\hline
\end{tabular}

TABLE 6 Algebraic index of convergence for European options observed by filter-COS $(T=0.025, T=0.1$ and $T=1)$.

\begin{tabular}{lcc}
\hline Filter & Put & Digital \\
\hline Fejér & 1 & 1 \\
Lanczos & 2 & 2 \\
Raised cosine & 2 & 2 \\
\hline Exponential $(p=2,4,6)$ & $p$ & $p$ \\
Vandeven $(p=1,2,3,4,5)$ & $p$ & $p$ \\
Erfc-Log $(p=1,2,3,4,5)$ & $p$ & $p$ \\
\hline
\end{tabular}

Figure 4 on the facing page displays the error of the COS formula for the option value and the Greeks for expiration time $T=0.1$ in a $\log -\log$ plot. Exponential filters with different orders are used. Note that for some cases the so-called roundoff-plateau, with minimal attainable accuracy due to machine precision, is reached. The use of filters improves the error and convergence order significantly, especially regarding the option Gamma $\Gamma$.

\subsubsection{Bermudan option price under VG}

A Bermudan-style option can be exercised at a set of $\mathcal{M}$ early-exercise dates prior to the expiration time $T, t_{0}<t_{1}<\cdots<t_{m}<\cdots<t_{\mathcal{M}}=T$, with timestep $\Delta t:=t_{m+1}-t_{m}$. The authors in Fang and Oosterlee (2009) describe a recursive algorithm, based on the COS method, for pricing Bermudan options backwards in time via Bellman's principle of optimality. We also employ the COS method for Bermudan options here, but replace the coefficients $\Phi_{k}$ by the filtered version, ie, by $\hat{s}(k / N) \Phi_{k}$, as in (3.18).

In Table 7 on page 100 and in Figure 5 on page 101 the results for the exponential filter with $T=1$ and different numbers of early-exercise dates are presented. The COS method with filtering becomes more and more beneficial when more early-exercise 
FIGURE 4 Error convergence for European options; put (top) and digital call (bottom) plus the Greeks, with exponential filters $(T=0.1)$.

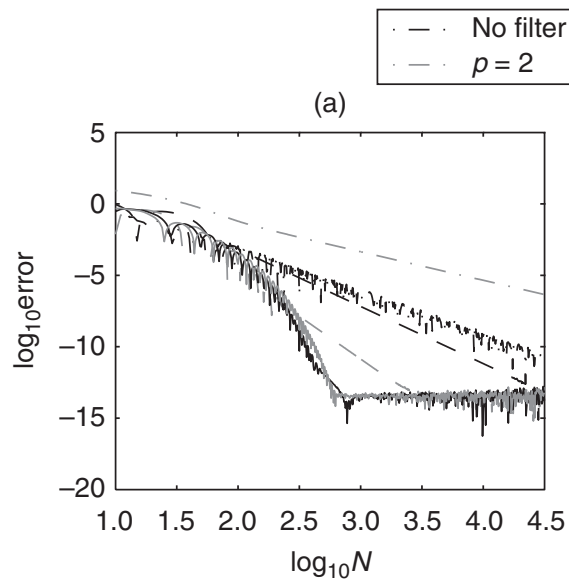

(b)
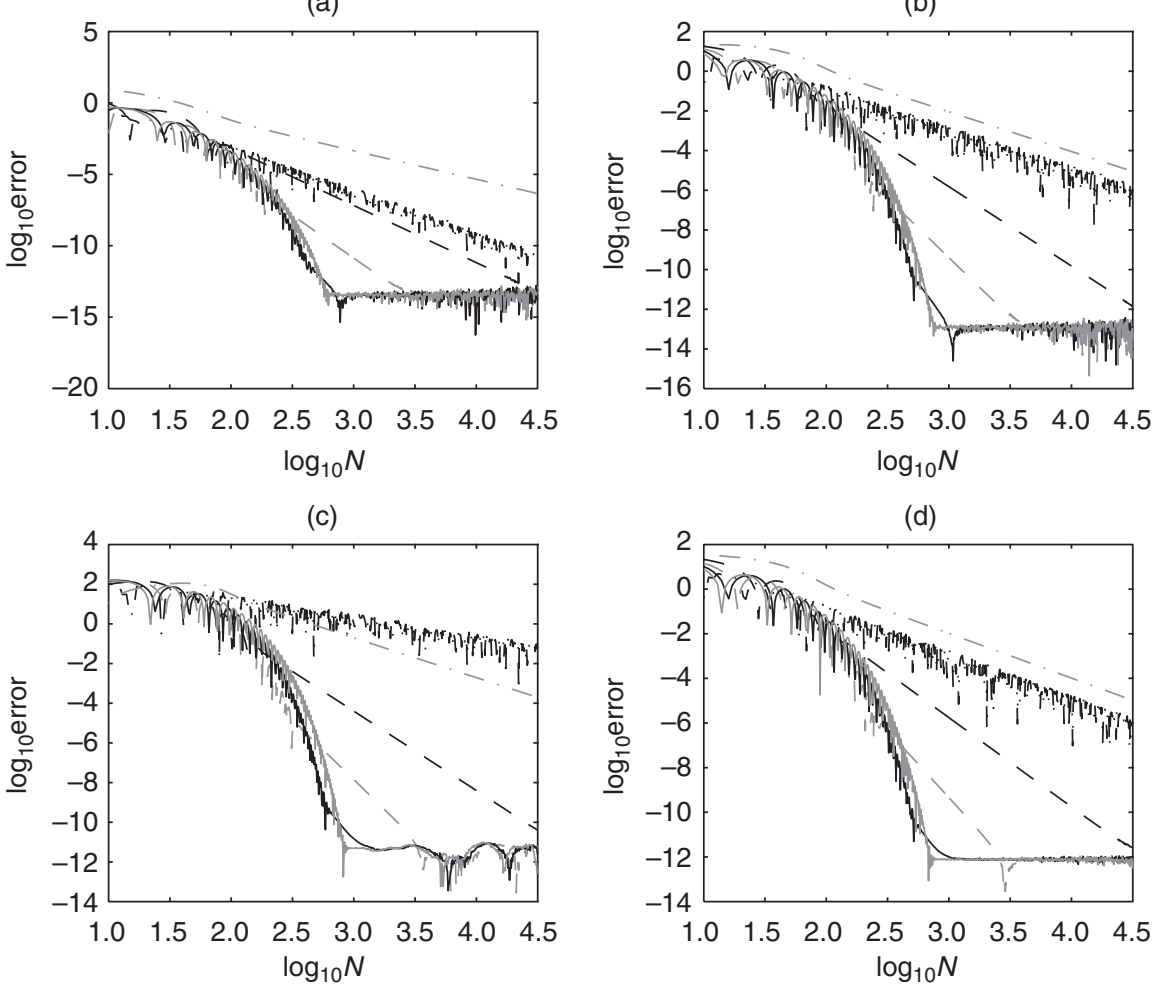

(d)

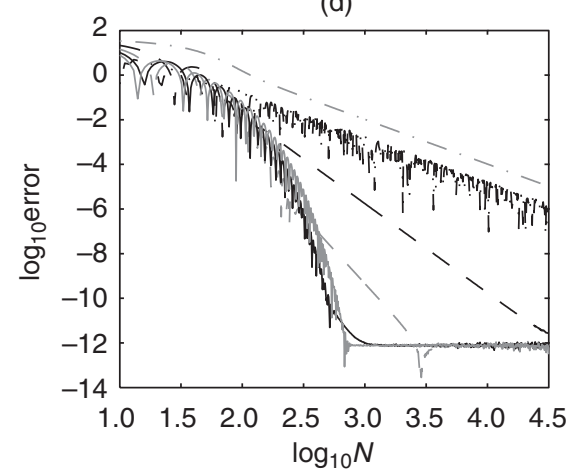

(e)
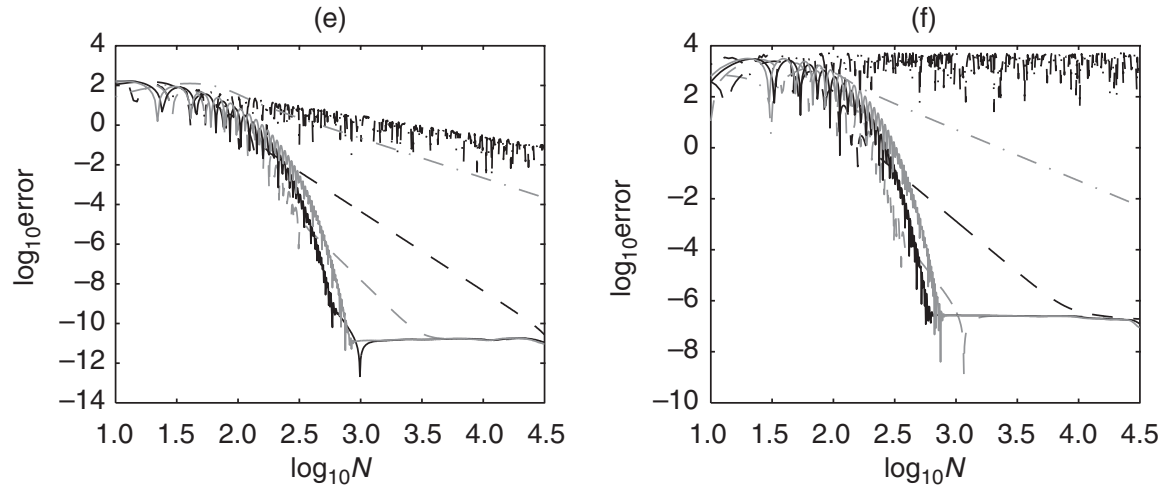

(a) Put. (b) Put Delta. (c) Put Gamma. (d) Digital call. (e) Digital call Delta. (f) Digital call Gamma. 
TABLE 7 Algebraic index of convergence for Bermudan put options with the filter-COS $\operatorname{method}(T=1)$.

\begin{tabular}{lccc}
\hline Filter & $\mathcal{M}=\mathbf{2}$ & $\mathcal{M N}=\mathbf{4}$ & $\mathcal{N}=\mathbf{8}$ \\
\hline No filter & 7 & 4.5 & 3.25 \\
\hline Fejér & 1 & 1 & 1 \\
Lanczos & 2 & 2 & 2 \\
Raised cosine & 2 & 2 & 2 \\
\hline Exponential $(p=2,4)$ & $p$ & $p$ & $p$ \\
Exponential $(p=6)$ & 6 & 6 & $*$ \\
Vandeven $(p=1,2,3,4,5)$ & $p$ & $p$ & $p$ \\
Erfc-Log $(p=1,2,3,4,5)$ & $p$ & $p$ & $p$ \\
\hline
\end{tabular}

dates are used, for example $\mathcal{M}=20$. Then the algebraic convergence rates are, however, not clearly measurable. A larger number of exercise dates implies a smaller timestep $\Delta t$ between the exercise dates, which decreases the convergence rate of the COS formula without filtering.

\subsection{Portfolio loss distribution}

In this section we present a final example, which has a financial background in risk management. The distribution function of interest will be a staircase function, which is difficult to approximate by Fourier series. The filtering technique will however improve the results significantly.

For a bank it is important to manage the risk originating from its business activities. The credit risk underlying a credit portfolio is one of the largest risk portions of a bank. For quantifying losses in credit portfolios one often looks at the value-at-risk (VaR). The VaR of a portfolio at confidence level $\alpha$ is given by the smallest value $x$ for which the probability that loss $L$ exceeds $x$ is at most $(1-\alpha)$ :

$$
\operatorname{VaR}_{\alpha}=\inf \{x \in \mathbb{R}: \mathbb{P}(L>x) \leqslant(1-\alpha)\}=\inf \left\{x \in \mathbb{R}: F_{L}(x) \geqslant \alpha\right\},
$$

where $F_{L}$ is the cumulative loss distribution function.

The Vasicek model (Vasicek 2002) is often used to find an approximation to the loss distribution and to compute the VaR. Under this model losses occur when an obligor defaults in a fixed time horizon. Suppose there are $m=1, \ldots, M$ issuers and $X_{m}$ represents the individual asset return of issuer $m$. In accordance with the Vasicek model we then use

$$
X_{m}=\sqrt{\rho_{m}} Y+\sqrt{1-\rho_{m}} Z_{m}, \quad m=1, \ldots, M,
$$


FIGURE 5 Error convergence for Bermudan options with an increasing number of earlyexercise dates, filter-COS technique with exponential filters $(T=1)$.

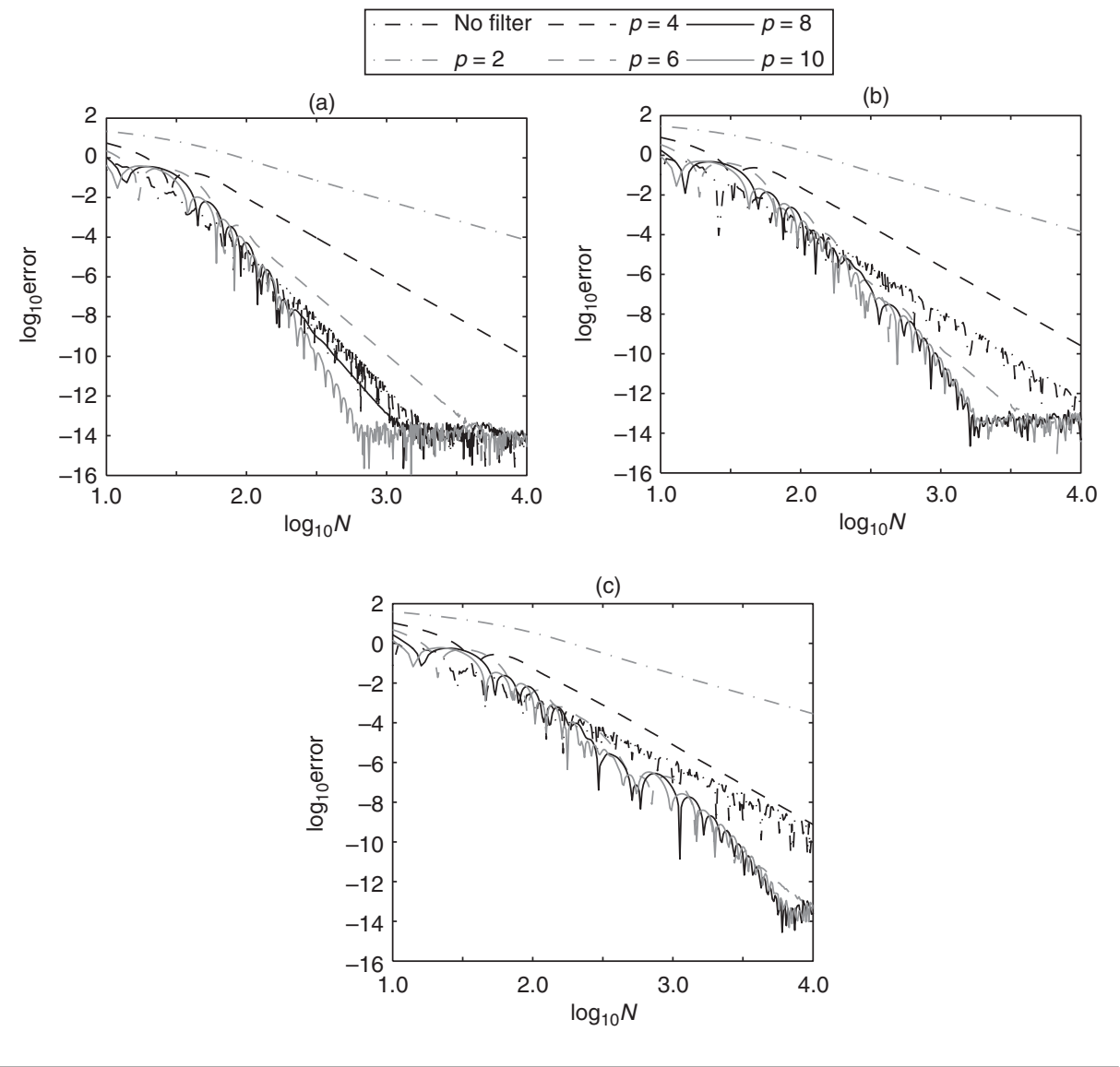

(a) $\mathcal{M}=2, v\left(t_{0}, x\right)=0.64386$. (b) $\mathcal{M}=4, v\left(t_{0}, x\right)=0.71161$. (c) $\mathcal{M}=8, v\left(t_{0}, x\right)=0.75220$.

where $Y$ is a common economic factor, $Z_{m}$ is the idiosyncratic factor for issuer $m$ and $\rho_{m}$ is the correlation between $Y$ and $Z_{m}$. All random variables are assumed to follow a standard normal distribution and $Y$ and $Z_{m}$ are independent. When the asset return falls below a default threshold $c_{m}$, there is a loss $\lambda_{m}$. We define the default probability of issuer $m$ by $P_{m}:=\mathbb{P}\left(X_{m}<c_{m}\right)$. The individual credit loss is defined by $L_{m}=\lambda_{m} \mathbf{1}_{X_{m}<c_{m}}$ and the total portfolio loss reads $L=\sum_{m=1}^{M} L_{m}$. If there is only one issuer then the total loss is given by the discrete random variable $\lambda_{1} \mathbf{1}_{X_{1}<c_{1}}$.

The COS method can be used to approximate the cumulative distribution function of the loss by means of its characteristic function, see (2.15), and thus to calculate 
the VaR. The probability distribution is based on a discrete set of events, resulting in a stepwise distribution function, which causes the COS method to suffer from the Gibbs phenomenon: with a loss distribution which is discontinuous, significant errors appear around the points of discontinuity (Fang 2012).

Following Masdemont and Ortiz-Gracia (2014) we take $M=20$ issuers, with default probability $P_{m}$ of $1 \%$, asset correlation $\rho_{m}$ of $50 \%$ and exposure $\lambda_{m}=1$. For the COS method we take $N=2^{10},[a, b]=\left[0, \sum_{m=1}^{M} \lambda_{m}\right]$. The characteristic function of $L$ can be written as

$$
\varphi(u)=\mathbb{E}\left[\mathrm{e}^{\mathrm{i} u L}\right]=\mathbb{E}\left[\mathbb{E}\left[\exp \left(\mathrm{i} u \sum_{m=1}^{M} \lambda_{m} \mathbf{1}_{X_{m}<c_{m}}\right) \mid Y\right]\right] .
$$

An analytic expression is available for the inner conditional expectation. In Fang (2012) an integration rule to approximate the outer expectation is employed, whereas in Glasserman and Ruiz-Mata (2006) Monte Carlo simulations on $Y$ were used. We test both approaches, with a grid, $y=[-5: 0.1: 5]$, for the numerical integration (denoted by COS $+\mathrm{NI}$ ) and by 5000 simulated values for $Y$ in the Monte Carlo experiment (denoted by COS $+\mathrm{MC}$ ). With these choices, the computation of the characteristic function, which is the most time-consuming part, is approximately 50 times more expensive for COS $+\mathrm{MC}$.

The loss distribution of this example portfolio is plotted in Figure 6 on the facing page. An accurate estimation of $1-F_{L}(L)$ results in an accurate VaR. The black dashed-dotted line is the result of a full Monte Carlo simulation, with 100000000 replications for each $X_{m}$, and serves as our reference solution here. The light-gray solid and gray dash-dotted lines are the COS method approximations with numerical integration and Monte Carlo simulation for $Y$, respectively. The black solid and black dashed lines are the filtered-COS results, using an exponential filter with $p=6$. The COS method without filtering shows a highly oscillatory behavior and does not give accurate results in the tail, where $1-F_{L}(L)$ is very small. The COS method with filter and with numerical integration, however, gives highly accurate results that correspond very well to the full Monte Carlo simulation. The difference gets smaller when the number of Monte Carlo simulations is increased. The results with the filter and Monte Carlo simulations for $Y, \mathrm{COS}+\mathrm{MC}$, are sensitive to outliers of draws for $Y$ and these results are not very satisfactory. We would also like to mention that approximations based on Haar wavelets (Masdemont and Ortiz-Gracia 2014; Ortiz-Gracia and Oosterlee 2013) give accurate portfolio loss VaR estimates as well.

REMARK 4.1 The model and computational technique can be extended to higherdimensional systematic risk factors $Y=\left[Y_{1}, \ldots, Y_{d}\right]$. In that case, the computation of the outer expectation in (4.10) can be performed by adaptive integration, as in Huang (2009). 
FIGURE 6 Recovery of portfolio loss distribution function using COS method and filtered version (exponential filter, order $p=6$ ).

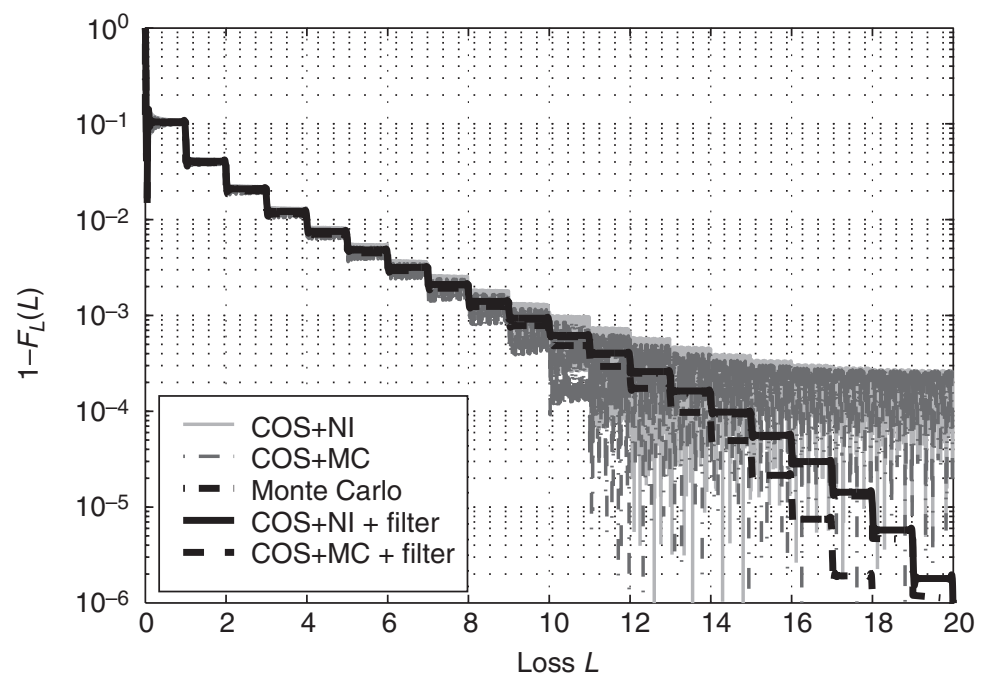

\section{CONCLUSIONS}

The COS method is an option pricing method based on Fourier cosine expansions which performs very well in general. When the underlying density function is smooth, we achieve an exponential convergence in the number of cosine coefficients. When the underlying density is not smooth, however, the method may suffer from the Gibbs phenomenon and the convergence is only of algebraic order. A filtering technique to improve the convergence rate for these cases has been discussed in the present paper. In practical cases where the COS method degrades due to discontinuities in functions, the convergence with a filter improves significantly in terms of the number of required Fourier coefficients as well as in CPU time.

Nonadaptive spectral filtering takes place in the Fourier domain and therefore combines very well with the COS option pricing formula, without adding significant computational costs. The Fourier coefficients are pre-multiplied by a decreasing function $\hat{s}(k / N)$ so that they decay faster and so that the convergence rate away from a discontinuity is improved. The technique can be used for one-dimensional problems, but also in higher dimensions.

In the numerical examples we tested six different filters, ie, the Fejér filter, the Lanczos filter, the raised cosine filter, the exponential filter, the Vandeven filter and 
the Erfc-Log filter. The exponential filter gave especially highly accurate results for stepwise functions.

The plain COS method for option pricing under the VG asset price process results in algebraic convergence. Our filter-COS formula improves the algebraic index of convergence, in particular for short time horizons. Moreover, for the computation of the option Greeks, which suffer from an even lower convergence rate without filtering, spectral filters are highly beneficial. As another example in finance, we discussed portfolio loss modeling. Discrete random variables then give rise to stepwise cumulative distribution functions. We derived a COS formula to recover the distribution, which, of course, also suffered from the Gibbs phenomenon and the resulting oscillations. The approximation drastically improves by applying spectral filters.

Improved convergence comes without additional computational costs in these applications and the fact that the filtering is easy to implement, even in multiple dimensions, makes the filter-COS method a natural solution for some of the problems described.

\section{DECLARATION OF INTEREST}

The authors report no conflicts of interest. The authors alone are responsible for the content and writing of the paper.

\section{ACKNOWLEDGEMENTS}

The authors wish to acknowledge F. Fang for sharing knowledge about the application of the COS method to portfolio loss modeling.

\section{REFERENCES}

Boyarchenko, S., and Levendorskii, S. (2011). New efficient versions of Fourier transform method in applications to option pricing. Preprint, SSRN 1846633.

Boyd, J. P. (1996). The Erfc-Log filter and the asymptotics of the Euler and Vandeven sequence accelerations. In Proceedings of the Third International Conference on Spectral and High Order Methods, A. V. Ilin and L. R. Scott (eds), pp. 267-276. Houston Journal of Mathematics, Houston, TX.

Boyd, J. P. (2001). Chebyshev and Fourier Spectral Methods. Courier Dover Publications, New York.

Boyd, J. P. (2011). A proof, based on the Euler sum acceleration, of the recovery of an exponential (geometric) rate of convergence for the Fourier series of a function with Gibbs phenomenon. In Spectral and High Order Methods for Partial Differential Equations, Hesthaven, J. S., and Rønquist, E. M. (eds). Lecture Notes in Computational Science and Engineering, Vol. 76, pp. 131-139. Springer.

Carr, P., and Madan, D. B. (1999). Option valuation using the fast Fourier transform. Journal of Computational Finance 2(4), 61-73. 
Chan, T. F., Osher, S., and Shen, J. (2001). The digital TV filter and nonlinear denoising. IEEE Transactions on Image Processing 10(2), 231-241.

Den Iseger, P. (2006). Numerical transform inversion using Gaussian quadrature. Probability in the Engineering and Informational Sciences 20(1), 1-44.

Duffie, D., Pan, J., and Singleton, K. J. (2000). Transform analysis and asset pricing for affine jump-diffusions. Econometrica 68(6), 1343-1376.

Fang, F. (2012). Use COS method to calculate portfolio credit loss. Internal report and personal communication.

Fang, F., and Oosterlee, C. W. (2008). A novel pricing method for European options based on Fourier-cosine series expansions. SIAM Journal on Scientific Computing 31(2), 826848.

Fang, F., and Oosterlee, C. W. (2009). Pricing early-exercise and discrete barrier options by Fourier-cosine series expansions. Numerische Mathematik 114(1), 27-62.

Fejér, L. (1900). Sur les Fonctions Bornées et Intégrables. Comptes Rendus, Paris, France.

Fusai, G., Marazzina, D., and Marena, M. (2011). Pricing discretely monitored Asian options by maturity randomization. SIAM Journal on Financial Mathematics 2(1), 383-403.

Gelb, A. (2000). A hybrid approach to spectral reconstruction of piecewise smooth functions. Journal of Scientific Computing 15(3), 293-322.

Glasserman, P., and Ruiz-Mata, J. (2006). Computing the credit loss distribution in the Gaussian copula model: a comparison of methods. Journal of Credit Risk 2(4), 33-66.

Gottlieb, D., and Shu, C. W. (1997). On the Gibbs phenomenon and its resolution. SIAM Review 39(4), 644-668.

Gut, A. (2005). Probability: A Graduate Course. Springer Texts in Statistics. Springer Science + Business Media.

Hesthaven, J. S., Gottlieb, S., and Gottlieb, D. (2007). Spectral Methods for Time-Dependent Problems. Cambridge Monographs on Applied and Computational Mathematics. Cambridge University Press.

Heston, S. L. (1993). A closed-form solution for options with stochastic volatility with applications to bond and currency options. Review of Financial Studies 6(2), 327-343.

Huang, X. (2009). Credit portfolio losses. Ph.D. Thesis, Delft University of Technology.

Lanczos, C. (1956). Applied Analysis. Prentice-Hall Mathematics Sries. Prentice-Hall.

Lord, R., and Kahl, C. (2007). Optimal Fourier inversion in semi-analytical option pricing. Journal of Computational Finance 10(4), 1-30.

Lord, R., Fang, F., Bervoets, F., and Oosterlee, C. W. (2008). A fast and accurate FFTbased method for pricing early-exercise options under Lévy processes. SIAM Journal on Scientific Computing 30(4), 1678-1705.

Madan, D. B., Carr, P., and Chang, E. C. (1998). The variance Gamma process and option pricing. European Finance Review 2(1), 79-105.

Masdemont, J. J., and Ortiz-Gracia, L. (2014). Haar wavelet-based approach for quantifying credit portfolio losses. Quantitative Finance 14(9), 1587-1595.

Ortiz-Gracia, L., and Oosterlee, C. W. (2013). Robust pricing of European options with wavelets and the characteristic function. SIAM Journal on Scientific Computing 35(5), B1055-B1084.

O'Sullivan, C. (2005). Path dependent option pricing under Lévy processes. European Finance Association 2005 Moscow Meetings Paper. 
Peyret, R. (2002). Spectral Methods for Incompressible Viscous Flow. Applied Mathematical Sciences, Vol. 148. Springer.

Ruijter, M. J., and Oosterlee, C.W. (2012). Two-dimensional Fourier cosine series expansion method for pricing financial options. SIAM Journal on Scientific Computing 34(5), B642B671.

Sarra, S. A. (2006). Digital total variation filtering as postprocessing for Chebyshev pseudospectral methods for conservation laws. Numerical Algorithms 41(1), 17-33.

Schoutens, W. (2003). Lévy Processes in Finance: Pricing Financial Derivatives. Wiley Series in Probability and Statistics. Wiley.

Stein, E. M., and Shakarchi, R. (2003). Fourier Analysis: An Introduction. Princeton Lectures in Analysis. Princeton University Press.

Surkov, V. (2009). Option pricing using Fourier space time-stepping framework. Ph.D. Thesis, University of Toronto.

Tadmor, E. (2007). Filters, mollifiers and the computation of the Gibbs phenomenon. Acta Numerica 16, 305-378.

Tadmor, E., and Tanner, J. (2005). Adaptive filters for piecewise smooth spectral data. IMA Journal of Numerical Analysis 25(4), 635-647.

Tanner, J. (2006). Optimal filter and mollifier for piecewise smooth spectral data. Mathematics of Computation 75(254), 767-790.

Vandeven, H. (1991). Family of spectral filters for discontinuous problems. Journal of Scientific Computing 6(2), 159-192.

van Slingerland, P., Ryan, J. K., and Vuik, C. (2011). Position-dependent smoothnessincreasing accuracy-conserving (SIAC) filtering for improving discontinuous Galerkin solutions. SIAM Journal on Scientific Computing 33(2), 802-825.

Vasicek, O. (2002). The distribution of loan portfolio value. Risk 15(12), 160-162.

Zhang, B., and Oosterlee, C. W. (2013). Efficient pricing of European-style Asian options under exponential Lévy processes based on Fourier cosine expansions. SIAM Journal on Financial Mathematics 4(1), 399-426. 